\title{
Web reviews: Musculoskeletal imaging
}

\author{
Surg Cdr IK Indrajit \\ Department of Radiodiagnosis and Imaging, Army Hospital (Research and Referral), Delhi Cantt - 110 010, India
}

Correspondence: Surg Cdr IK Indrajit, Army Hospital (Research and Referral), Delhi Cantt - 110010 , India. E-mail: inji63@gmail.com

Few useful websites dealing with musculoskeletal imaging and related topics are reviewed below.

1. A large collection of Plain Radiographs of Bony Skeleton is available at http://myweb.lsbu.ac.uk/dirt/museum/ skeletonanat.html. This site, featuring individual cases, has sections covering various parts of the body, including the limbs, chest wall, and general bone radiology. The site, available on-line since November 1994, has been created by Ian Maddison, from London South Bank University.

2. Dwarfism and dysplasia syndromes at http://www. stevensorenson.com/residents6/ makes use of an electronic teaching file to categorize dwarfism and the dysplasia syndromes. Authored by a team headed by Steven M Sorenson et al. from the UCLA School of Medicine and VA Greater Los Angeles Healthcare System, the site focuses on common conditions causing dwarfism and also deals with dysplasia syndromes.

3. Skeletal trauma is available at http://www.meded.virginia.edu/courses/rad/ext/index.html. It is sourced from the University of Virginia as a part of an interactive tutorial titled 'An introduction to radiology online' at http://www.med-ed.virginia.edu/courses/rad/. This selftutorial is useful for residents and medical students to assess radiographs in cases of skeletal trauma. Normal anatomy and a variety of fractures dealing with the hand, elbow, spine, pelvis, knee, ankle, foot, and heel are illustratively analyzed.

4. An outline of Musculoskeletal Ultrasound authored by John Lin and William Weadock from the University of Michigan is available at http://www.med.umich.edu/ $\mathrm{rad} / \mathrm{muscskel} / \mathrm{mskus} /$ index.html. The site comprises illustrative sections on technique and anatomy with examples and references. Similarly, Ultrasound of the shoulder is available at http://ej.rsna.org/EJ_0_96/000896/ustechnique.html. Featured sections include anatomy, technique, cuff tears, post-op, trauma, tendinitis, arthritis, and the biceps tendon.

5. European League Against Rheumatism (EULAR) is a society that focuses on research, prevention, treatment, and rehabilitation of rheumatic diseases and is available online at http://www.eular.org/. The 'Eular Guidelines for Musculoskeletal Ultrasound in Rheumatology' is available at http://www.doctor33.it/eular/ultrasound/ Guidelines.htm, authored by Marina Backhaus and
Peter Balint et al. and edited by Walter Grassi and Emilio Filippucci from the Clinica Reumatologica at Università di Ancona, Italy. The 'guidelines' has sections focusing on technical equipment, teaching, and training as well as standardization of musculoskeletal USG. The section on standardization has educative information on positioning of the patient and is illustrated with standard scans of the shoulder, elbow, wrist, hand, hip, knee, ankle, and foot.

6. Bonepit sourced from the Department of Musculoskeletal Radiology at the University of California, San Diego Medical Center (UCSD) is available at http://www. medicine.ucsd.edu/bonepit. The website offers a few useful and educative sections. One such is a ready reckoner of classification systems of various common musculoskeletal entities such as Hangman's fractures, SLAP tears of the shoulder, Garden type hip fractures and Lauge-Hansen ankle fractures. It is available at http://www.medicine.ucsd.edu/bonepit/Classifications/ Classifications\%20home.htm.

7. Bone tumor.org at http://www.bonetumor.org 'is an educational website for physicians and patients who are interested in learning about all the types of tumors and cancers that affect the skeletal system.' The site created by Henry DeGroot III and a dedicated team from the University of Massachusetts, is endowed with many sections like 'Case of the Month,' an online atlas of bone tumors and bone cancers, tumor mimics, and metastatic bone lesions.

8. Musculoskeletal Radiology at http://www.gentili. net/ comprises a set of webpages featuring the musculoskeletal system and is authored by A. Gentili, M.D from the UCSD Department of Radiology. The educative material is wide-ranging and the impressive topic list includes atlases on arthritides of the hand and foot, a clickable fracture atlas, and many lists of differential diagnosis. An interactive atlas of 'signs' is at hand at http://www.gentili.net/signs/. It can be searched by the name of the sign, the pathological diagnosis, and locations and features many signs used commonly in musculoskeletal radiology.

9. Approaches to Differential Diagnosis in Musculoskeletal Imaging is an introductory textbook of musculoskeletal imaging available online at http://www.rad.washington. edu/mskbook/. Authored by M. L. Richardson from the 
Department of Radiology, University of Washington, the contents includes educative topics like arthritis: appendicular and axial, lucent and sclerotic lesions of bone, periosteal reaction, fractures without significant trauma, the painful joint prosthesis orthopedic hardware, scoliosis, and skeletal dysplasias.

10. Wheeless' Textbook of Orthopedics available at http:// www.wheelessonline.com/ is a premier website hosted by the Duke University Medical Center's Division of Orthopedic Surgery, in conjunction with Data Trace Internet Publishing. It is a comprehensive online medical textbook, wherein nearly 11,000 pages with more than 5,000 images are available. Broad sections include arthritis, arthroscopy, bone, DVT, joints, muscles, tendon, nerves, trauma, and fractures. However, one can also make use of a search facility by word or by site index. Each topic is fully searchable by alphabetical, anatomical, and keyword searches or a click on the diagram of a whole body skeleton.

\section{End piece}

Traditionally, searches for radiology articles and citations on the web are commonly performed using Pubmed and other Medline-based programs or from webpages of specific journals. In recent times, there has been renewed interest in scientific searches, due to the emergence of sites like Google Scholar and Scirus. While collectively they are useful, a certain amount of heterogeneity exists in their yield of results, making their use an experience ranging from the dismal to the delightful.

Google Scholar is a fast and comprehensive search engine available at http://scholar.google.com/ for locating scholarly literature. Launched in end 2004, the main thrust of Google Scholar is that it searches 'many disciplines and sources: peer-reviewed papers, theses, books, abstracts, and articles from academic publishers, professional societies, preprint repositories, universities, and other scholarly organizations.' Additionally, it has convenient tools like 'Interface Language' and 'Scholar Preferences' that can be set to suit the users taste.

Scirus named after a Greek legendary figure, is a sciencespecific search engine available at http://www.scirus.com/ on the Internet. Started in 2001 by Elsevier, Scirus pinpoints 'scientific, scholarly, technical, and medical data on the Web' by focusing on content available 'in web information, preprint servers, digital archives, repositories, and patent and journal databases.' Scirus allows searches by author, journal, or article or by specified date ranges.

For those specifically interested in MR imaging, here is a site full of zip as well as actively updated contents. MR-TIP.com or Magnetic Resonance Technology Information Portal available at http://www.mr-tip.com/serv1.php?type $=\mathrm{db}$ is essentially a web portal having a large database of educative material with links to more than two thousand MRI-related publications and resource sites. The key features of this site include Info Sheets on topics such as MRI artifacts, coils, contrast agents, devices, and sequences, a Comparison of Vendor Acronyms at http://www.mr-tip.com/serv1. php?type $=$ cam, and MRI Resource Directory Overview at http://www.mr-tip.com/serv1.php?type=ldir.

Source of Support: Nil, Conflict of Interest: None declared. 